\title{
Mathematical Analysis of Magnetized Rotating Nanofluid Flow Over nonlinear shrinking surface: Duality and Stability
}

\author{
Sumera Dero ${ }^{1}$, Ghulam Hyder Talpur ${ }^{2}$, Abbas Ali Ghoto ${ }^{3}$, Shokat Ali $^{1}$
}

\begin{abstract}
:
In this study, the magnetohydrodynamic (MHD) effect on the boundary layer rotating flow of a nanofluid is investigated for the multiple branches case. The main focus of current research is to examine flow characteristics on a nonlinear permeable shrinking sheet. Moreover, the governing partial differential equations (PDEs) of the problem considered are reduced into coupled nonlinear ordinary differential equations (ODEs) with the appropriate similarity transformation. Numerical results based on the plotted graphs are gotten by solving ODEs with help of the three-stage Labatto IIIA method in bvp4c solver in MATLAB. To confirm numerical outcomes, current results have been compared with previously available outcomes and found in good agreement. Skin friction coefficients, Nusselt and Sherwood numbers, velocity profiles, temperature profiles, and concentration profiles are examined. The results show that dual (no) branches exist in certain ranges of the suction parameter i.e., $S \geq S_{c}\left(S<S_{c}\right)$. Further, profiles of velocity decrease for rising values of Hartmann number in the upper branch, while a reverse trend has been noticed in the lower branch. Profiles of temperature and concentration enhance for the increasing values of thermophoresis in both branches. stability analysis of the branches is also done and noticed that the upper branch is a stable branch from both branches. Finally, it is noted that the stable branch has symmetrical behavior with regard to the parameter of rotation.
\end{abstract}

Keywords: 3D flow; nanofluid; Rotating shrinking surface; Dual Branches; Stability analysis.

\section{Introduction}

Recently, scholars are implicated in the analysis of rotational flows within stretching and shrinking boundary layer problems because of their widespread use in the system of rotor-stator, food processing, spinning devices, the architecture of gas turbines, disk cleaners, and many others. Wang [1] examined the flow of rotating fluid through the stretching sheet where momentum boundary layer thickness was observed to decrease as the parameter of the rotational impact increased. Takhar et al. [2] considered a rotating fluid flow on the stretching surface with the characteristics of a magnetic number. Shafique et al. [3] investigated the rotating effect in the Maxwell fluid by considering binary chemical reactions and energy activation characteristics. They have found that the hydrodynamic boundary layer thins when rotation parameter $\lambda$ is incremented. Oscillatory behavior in both $x$ - and $y$-components of velocity is observed when rotation parameter $\lambda$ is sufficiently large. Rashad [4] used mathematical modeling to check the effect of the non-steady MHD flow of rotating fluid. Recently, Ullah et al. [5] found that

${ }^{1}$ Institute of Mathematics and Computer Science, University of Sindh, Jamshoro, Pakistan.

${ }^{2}$ Department of Statistics, University of Sindh, Jamshoro 76080, Pakistan.

${ }^{3}$ Department of Mathematics and Statistics, Quaid-e-Awam University, Pakistan. Corresponding Author: sumera.dero@usindh.edu.pk 
Sumera Dero (et al.), Mathematical Analysis of Magnetized Rotating Nanofluid Flow Over nonlinear shrinking surface:

temperature and concentration were increasing functions of the porosity and the Forchheimer parameters during the examination of the rotating flow of the nanofluid. In addition, Hayat et al. [6] extended the rotational flow model to examine the characteristics of homogeneous-heterogeneous nanofluid reactions. Lund et al., [7] reviewed the 3D flow of rotating nano-fluid on an exponential plane and discovered that the solution was not unique when the value of the rotating parameter was less than 0.1. Some important effects of the various physical parameter on the rotating flow can be found in [7-12].

The influence of MHD has attracted a lot of attention from researchers because of its extensive range of uses in various fields of science. It was introduced by Hannes Alfvén (1908-1995) who was a famous Swedish physicist. In 1970, he received the Physics Nobel prize for his pioneering work in MHD and major applications in numerous portions of plasma physics. In general, the presence of the magnetic field and the electrically conductive flow of the fluid give rise to the induced electrical current. More interest in MHD flow began in 1930 when Hartmann invented an electromagnetic pump. After that, many researchers considered MHD in their studies such as William [13-14], Eastman et al. [15], and Hossain [16] due to its extensive uses. Moreover, magnetic fields have an impact on numerous artificial and natural flows, which are essential elements used in some industries like pumping, heating, and levitating metals in the core of the earth. For example, solar flares and sunspots are generated by the solar magnetic field [17-18]. For medical and other applications, the ideal properties of the finished product are determined by drawing these strips or filaments into an electrically conductive liquid under the influence of a magnetic field. Hsiao [19] investigated numerical solutions for MHD two-dimension steady flow of boundary layer in a micropolar nanofluid. The author considered Buongiorno's model [20] with a viscous dissipation effect on a linear non-permeable stretching surface. The linear non-permeable stretching surface was used because only one solution was considered. Further, it was discovered that an upsurge in the magnetic field decreases in the magnitudes of Nusselt number and velocities. Recently, Dero et al. [21] used the MHD effect on nanofluid of micropolar where the effect of the thermal radiation had been studied. The shooting method was then adopted to solve the resultant ODEs and triple solutions were obtained. Some important effects of the MHD on fluid flow can be seen in these articles [22-28].

In view of the development of new technologies over the last few decades, the use of convective fluids for heat transfer, such as oil, ethylene glycol, and water minerals, has increased significantly. These kinds of fluids are an essential part of numerous industrial sectors including air-conditioning, transportation, and power generation [29]. It seems that these convection fluids could not meet the requirements of the rate of heat transfer and cooling. In this regard, different fluid upgrade procedures have been applied as there is a necessity to make novel kinds of fluid that are extra viable in relation to heat transfer act to meet the increasing demands of modern technology and innovation in miniaturization and process intensification of equipment [30]. Keeping in mind the final goal to attain such, it has recently been anticipated to mix insignificant amounts of nanometers from 10 $\mathrm{nm}$ to $50 \mathrm{~nm}$ of nanoparticles in convectional fluids, subsequent in nanofluids [31-33]. Studies have shown that the fraction of particle volume, that is the concentration of volumetric of the nanoparticle in nanofluid is related to the nanofluid thermal conductivity [34-35]. As associated with convectional fluid, the results of experiments on nanofluid noted that the expressively of thermal conductivity expanded with little changes in nanoparticle volume fractions. The nanofluids' thermal conductivity with the base fluid of water containing nanoparticles $\mathrm{TiO}_{2}(27 \mathrm{~nm}), \mathrm{SiO}_{2}$ $(12 \mathrm{~nm})$, and $\mathrm{Al}_{2} \mathrm{O}_{2}(13 \mathrm{~nm})$ have been measured by Masuda et al. [36]. Abareshi et al. [37] and Das et al. [38] suggested that there is a significant rise in temperature with an increase in thermal conductivity. $\mathrm{CuO}$ (28.6 $\mathrm{nm}) /$ water and $\mathrm{Al}_{2} \mathrm{O}_{2}(38.4 \mathrm{~nm}) /$ water nanofluids at different temperatures fluctuating from $21^{\circ} \mathrm{C}$ to $51^{\circ} \mathrm{C}$ were studied by 
Sumera Dero (et al.), Mathematical Analysis of Magnetized Rotating Nanofluid Flow Over nonlinear shrinking surface: Duality and Stability (pp. $01-13)$

Das et al. [39]. Theoretically, due to an increase in Brownian motion along with nanoparticles and nanofluid's bulk temperature $T$, it is expected that more energy can be exchanged from one region to the next as time increased.

The flow of boundary layer idea on an incessant stretched sheet along constant velocity was initially presented by Sakiadis [40-41]. Ever since, frequent research on boundary layer flow through a stretched sheet had been carried out because of its extensive applications in industries such as the production of glass fiber, hot rolling, paper production, and polymer sheets extrusion [42]. Crane [43] was the first who consider a fluid flow problem on a stretched surface and presented a solution in an analytical form. In recent years, Awaludin et al. [44] investigated heat transfer of a stagnation point flow with the effect of heat sink/source on a stretched/shrunk parameter. They observed that dual solutions existed indefinite ranges of stretching/shrinking parameters. Recently, Raza et al. [45] studied the three-dimensional boundary layer flow of a rotating nanofluid in the presence of suction/injection. The numerical results revealed two solutions existed which depended on the values of magnetic, stretching/shrinking, porosity, and suction parameters.

Based on the literature review conducted and published, there is no such study in which a rotational nanofluid model with Brownian motion and thermophoresis effect on the stretching surface has been considered for multiple branches with their stability analysis. Due to this research gap, this study is conducted numerically as the nanofluid rotational flow has important applications in industry, engineering, and so on. In light of this fact, the Buongiorno model was considered to be preparing a nanofluid model in the hope that our findings will provide valuable help and reduce the cost of experiments.

\section{Mathematical Formulation}

The steady three-dimensional flow of nanofluid along with heat transfer has been considered over the shrinking surface as presented in Figure 1. Sheet at $z=0$ in the direction of $x$-axis i.e, $u_{w}(x)=-c x^{n}$ and velocity of mass flux is $w_{w}(x)=$ $S \sqrt{c \vartheta} x^{(n-1) / 2}$. Moreover, the temperature at the wall (ambient) is $T_{w}\left(T_{\infty}\right)$. The sheet is supposed to rotate with velocity $\Omega_{0}$ about the $Z$-axis that is vertical to the sheet. A uniform field of external magnetic $B=B_{0}$ has been used to act with the $\mathrm{z}$-axis. Taking into account the momentum, temperature, and concentration boundary layers, the flow of nanofluid can be presented in the form of PDEs as follows [45]:

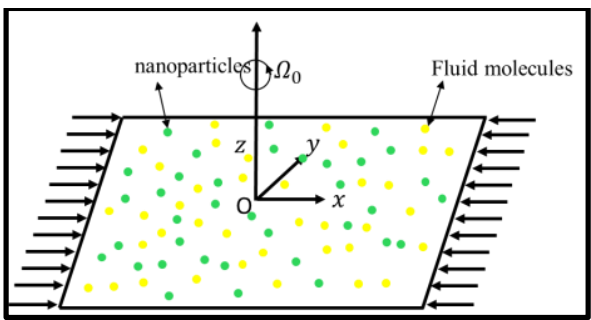

Figure 1. Physical model.

$$
\begin{aligned}
& \frac{\partial u}{\partial x}=-\left(\frac{\partial w}{\partial z}+\frac{\partial v}{\partial y}\right) \\
& u \frac{\partial u}{\partial x}-2 \Omega_{0} v=\vartheta \frac{\partial^{2} u}{\partial z^{2}}-\frac{\sigma B^{2} u}{\rho}-v \frac{\partial u}{\partial y} \\
& -w \frac{\partial u}{\partial z} \\
& u \frac{\partial v}{\partial x}+2 \Omega_{0} u=\vartheta \frac{\partial^{2} v}{\partial z^{2}}-\frac{\sigma B^{2} v}{\rho}-v \frac{\partial v}{\partial y} \\
& -w \frac{\partial v}{\partial z} \\
& u \frac{\partial T}{\partial x}-\alpha \frac{\partial^{2} T}{\partial z^{2}}=-v \frac{\partial T}{\partial y}-w \frac{\partial T}{\partial z}+ \\
& \tau_{1}\left[D_{B} \frac{\partial C}{\partial z} \frac{\partial T}{\partial z}+\frac{D_{T}}{T_{\infty}}\left(\frac{\partial T}{\partial z}\right)^{2}\right] \\
& u \frac{\partial C}{\partial x}+v \frac{\partial C}{\partial y}+w \frac{\partial C}{\partial z}=D_{B} \frac{\partial^{2} C}{\partial z^{2}}+ \\
& \frac{D_{T}}{T_{\infty}} \frac{\partial^{2} T}{\partial z^{2}}
\end{aligned}
$$
5) are
The related boundary conditions (BCs) (1-

Sukkur IBA Journal of Computing and Mathematical Science - SJCMS | Vol. 5 No. 2 July - Dec 2021 
Sumera Dero (et al.), Mathematical Analysis of Magnetized Rotating Nanofluid Flow Over nonlinear shrinking surface:

$$
\left\{\begin{array}{c}
v=w_{w}(x), u=u_{w}(x), v=0, \text { at } z=0 \\
T=T_{w}, C=C_{w} \text { at } z=0 \\
u \rightarrow 0, v \rightarrow 0, T \rightarrow T_{\infty}, \text { as } z \rightarrow \infty \\
C \rightarrow C_{\infty} \text { as } z \rightarrow \infty
\end{array}\right.
$$

Note that respective velocities in directions of $x, y$, and $z$-axes are $u, v$, and $w$. Further, $\vartheta, \sigma, \alpha, T, \tau_{1}, D_{B}, D_{T}$, and $C$ are the corresponding kinematics viscosity, electrical conductivity, thermal diffusivity, temperature, the ratio between heat capacitances of the nanoparticles and base fluid, Brownian diffusion, thermophoresis diffusion, and nanoparticle fraction or concentration of nanofluid.

Lund et al. [46]'s similarity variables are employed as follows:

$$
\left\{\begin{array}{c}
u=c x^{n} f^{\prime}(\eta), v=c x^{n} g(\eta) \\
w=-\sqrt{\frac{c \vartheta(n+1)}{2}} x^{(n-1) / 2} \\
{\left[f+\frac{n-1}{n+1} \eta f^{\prime}\right]} \\
\eta=z \sqrt{\frac{c(n+1)}{2 \vartheta} x^{(n-1) / 2}} \\
\emptyset(\eta)=\left(C-C_{\infty}\right) /\left(C_{w}-C_{\infty}\right) \\
\theta(\eta)=\left(T-T_{\infty}\right) /\left(T_{w}-T_{\infty}\right)^{\prime}
\end{array}\right.
$$

where prime shows the derivative with respect to $\eta$ and $c$ is a positive constant. By putting Equation (7) in (2-5) leads to

$$
\begin{gathered}
f^{\prime \prime \prime}+f f^{\prime \prime}-\frac{2 n}{n+1} f^{\prime 2}+\frac{4 \Omega}{n+1} g \\
-\frac{2 n}{n+1} M f^{\prime}=0 \\
g^{\prime \prime}+f g^{\prime}-\frac{2 n}{n+1} f^{\prime} g-\frac{4 \Omega}{n+1} f^{\prime} \\
-\frac{2 n}{n+1} M g=0 \\
\frac{1}{P r} \theta^{\prime \prime}+\theta^{\prime} f+N b \emptyset^{\prime} \theta^{\prime}+N t\left(\theta^{\prime}\right)^{2} \\
=0 \\
\emptyset^{\prime \prime}+S c f \emptyset^{\prime}+\frac{N t}{N b} \theta^{\prime \prime}=0
\end{gathered}
$$

Along with BCs

$$
\left\{\begin{array}{c}
f^{\prime}(0)=-1, \theta(0)=1, \emptyset(0)=1 \\
f(0)=-S \sqrt{\frac{2}{n+1}} \\
g(\eta) \rightarrow 0, f^{\prime}(\eta) \rightarrow 0 \text { as } \eta \rightarrow \infty \\
\theta(\eta) \rightarrow 0, \emptyset(\eta) \rightarrow 0 \text { as } \eta \rightarrow \infty
\end{array}\right.
$$

Here $\Omega=\frac{\Omega_{0}}{c}$ is rotation parameter, $\operatorname{Pr}=$ $\frac{\vartheta_{f}}{\alpha_{f}}$ is Prandtl, $N b=\frac{\tau_{1} D_{B}\left(C_{w}-C_{\infty}\right)}{\vartheta}$ is Brownian motion parameter, $N t=\frac{\tau_{1} D_{T}\left(T_{W}-T_{\infty}\right)}{\vartheta T_{\infty}} \quad$ is thermophoresis parameter, and $S$ is the injection parameter $(S>0)$ and suction parameter $(S<0)$.

The skin friction coefficients, local Nusselt, and Sherwood numbers can be defined as

$$
\left\{\begin{array}{c}
C_{f x}=\frac{\mu}{\rho u_{w}^{2}}\left(\frac{\partial u}{\partial z}\right) \mid z=0 \\
C_{f y}=\frac{\mu}{\rho v_{w}^{2}}\left(\frac{\partial v}{\partial z}\right) \mid z=0 \\
N u_{x}=-\frac{x}{\left(T_{w}-T_{\infty}\right)}\left(\frac{\partial T}{\partial z}\right) \mid z=0 \\
S h_{x}=-\frac{x}{\left(C_{w}-C_{\infty}\right)}\left(\frac{\partial C}{\partial z}\right) \mid z=0
\end{array}\right.
$$
gives

Putting Equation (7) in Equation (13) $\left\{\begin{array}{l}\sqrt{R e_{x}} C_{f x}=\frac{n+1}{2} f^{\prime \prime}(0) \\ \sqrt{R e_{y}} C_{f y}=\frac{n+1}{2} g^{\prime}(0) \\ \sqrt{\frac{1}{R e_{x}}} N u_{x}=-\theta^{\prime}(0), \\ \sqrt{\frac{1}{R e_{x}}} S h_{x}=-\emptyset^{\prime}(0)\end{array}\right.$

where $R e_{x}=\frac{x u_{w}}{\vartheta}$ and $R e_{y}=\frac{y v_{w}}{\vartheta}$ are the local Reynold numbers. 
Sumera Dero (et al.), Mathematical Analysis of Magnetized Rotating Nanofluid Flow Over nonlinear shrinking surface: Duality and Stability

\section{Temporal Stability Analysis}

In the previous section, dual solutions of Equations (8-11) with BCs (12) are noted; these branches are important as BCs (12) are also fulfilled in the lower branch. It is critical, however, to obtain a solution that can maintain its stability when subjected to minor disturbances. The stability of two solutions is so evaluated in order to identify a branch that is perfectly appropriate to the actual natural situation. The first step for stability is to transform Eqs (2-5) to the unsteady form as per the stability criteria as follows [47]:

$$
\begin{gathered}
\frac{\partial u}{\partial t}+u \frac{\partial u}{\partial x}-2 \Omega_{0} v=\vartheta \frac{\partial^{2} u}{\partial z^{2}}-\frac{\sigma B^{2} u}{\rho}- \\
v \frac{\partial u}{\partial y}-w \frac{\partial u}{\partial z} \\
\frac{\partial v}{\partial t}+u \frac{\partial v}{\partial x}+2 \Omega_{0} u=\vartheta \frac{\partial^{2} v}{\partial z^{2}}-\frac{\sigma B^{2} v}{\rho}- \\
v \frac{\partial v}{\partial y}-w \frac{\partial v}{\partial z} \\
\frac{\partial T}{\partial t}+u \frac{\partial T}{\partial x}-\alpha \frac{\partial^{2} T}{\partial z^{2}}=-v \frac{\partial T}{\partial y}-w \frac{\partial T}{\partial z}+ \\
\tau_{1}\left[D_{B} \frac{\partial C}{\partial z} \frac{\partial T}{\partial z}+\frac{D_{T}}{T_{\infty}}\left(\frac{\partial T}{\partial z}\right)^{2}\right] \\
\frac{\partial C}{\partial t}+u \frac{\partial C}{\partial x}+v \frac{\partial C}{\partial y}+w \frac{\partial C}{\partial z}=D_{B} \frac{\partial^{2} C}{\partial z^{2}}+ \\
\frac{D_{T}}{T_{\infty}} \frac{\partial^{2} T}{\partial z^{2}}
\end{gathered}
$$

where $t$ indicates the time. As a result, a new variable, $\tau=c x^{n-1} t$, is established. Equation (7) is articulated as follows:

$$
\left\{\begin{array}{c}
u=c x^{n} f^{\prime}(\eta, \tau), v=c x^{n} g(\eta, \tau) \\
w=-\sqrt{\frac{c \vartheta(n+1)}{2}} x^{(n-1) / 2} \\
{\left[f+\frac{n-1}{n+1} \eta f^{\prime}\right], \tau=c x^{n-1} t} \\
\eta=z \sqrt{\frac{c(n+1)}{2 \vartheta}} x^{(n-1) / 2} \\
\emptyset(\eta, \tau)=\left(C-C_{\infty}\right) /\left(C_{w}-C_{\infty}\right) \\
\theta(\eta, \tau)=\left(T-T_{\infty}\right) /\left(T_{w}-T_{\infty}\right)
\end{array}\right.
$$

Substituting Equation (19) in Equations (15-18) leads to

$$
\begin{aligned}
& f_{\eta \eta \eta}+f f_{\eta \eta}-\frac{2 n}{n+1} f_{\eta}^{2}+\frac{4 \Omega}{n+1} g- \\
& \frac{2 n}{n+1} M f_{\eta}-\frac{2 n}{n+1} f_{\tau \eta}=0 \\
& g_{\eta \eta}+f g_{\eta}-\frac{2 n}{n+1} f_{\eta} g-\frac{4 \Omega}{n+1} f_{\eta}- \\
& \frac{2 n}{n+1} M g-\frac{2 n}{n+1} g_{\tau}=0 \\
& \frac{1}{P r} \theta_{\eta \eta}+f \theta_{\eta}+N b \emptyset_{\eta} \theta_{\eta}+N t\left(\theta_{\eta}\right)^{2}- \\
& \theta_{\tau}=0 \\
& \emptyset_{\eta \eta}+S c f \emptyset_{\eta}+\frac{N t}{N b} \theta_{\eta \eta}-S c \emptyset_{\tau}=0
\end{aligned}
$$

Along with BCs

$$
\left\{\begin{array}{c}
f^{\prime}(0, \tau)=-1, \theta(0, \tau)=1, \emptyset(0, \tau)=1 \\
f(0, \tau)=-S \sqrt{\frac{2}{n+1}} \\
g(\eta, \tau) \rightarrow 0, f^{\prime}(\eta, \tau) \rightarrow 0 \text { as } \eta \rightarrow \infty \\
\theta(\eta, \tau) \rightarrow 0, \emptyset(\eta, \tau) \rightarrow 0 \text { as } \eta \rightarrow \infty
\end{array}\right.
$$

Now, obtain the solutions of steady flow from (8-11) as $f(\eta)=f_{0}(\eta), g(\eta)=g_{0}(\eta)$, $\theta(\eta)=\theta_{0}(\eta)$, and $\phi(\eta)=\emptyset_{0}(\eta), \quad$ it is assumed

$$
\left\{\begin{array}{l}
f(\eta, \tau)=f_{0}(\eta)+e^{-\varepsilon \tau} F(\eta, \tau) \\
g(\eta, \tau)=g_{0}(\eta)+e^{-\varepsilon \tau} G(\eta, \tau) \\
\theta(\eta, \tau)=\theta_{0}(\eta)+e^{-\varepsilon \tau} H(\eta, \tau) \\
\varnothing(\eta, \tau)=\emptyset_{0}(\eta)+e^{-\varepsilon \tau} J(\eta, \tau)
\end{array}\right.
$$


Sumera Dero (et al.), Mathematical Analysis of Magnetized Rotating Nanofluid Flow Over nonlinear shrinking surface:

where the unidentified eigenvalue is $\varepsilon$ where its value needs to fix a stable branch. In addition, $F(\eta, \tau), G(\eta, \tau), H(\eta, \tau)$, and $J(\eta, \tau)$ all their derivatives are assumed small relative to $f_{0}(\eta), g_{0}(\eta), \theta_{0}(\eta)$, and $\emptyset_{0}(\eta)$. Now, substituting the correlation (25) in Equations (20-24), we get the following resultant Linearized Eigenvalue Problem (LEVP) system as follows:

$F_{0}^{\prime \prime \prime}+f_{0} F_{0}^{\prime \prime}+F_{0} f_{0}^{\prime \prime}-\frac{4 n}{n+1} f_{0}^{\prime} F_{0}^{\prime}+\frac{4 \Omega}{n+1} G_{0}$

$\frac{2 n}{n+1} M F_{0}^{\prime}+\frac{2 n}{n+1} \varepsilon F_{0}^{\prime}=0$

$G_{0}^{\prime \prime}+g_{0}^{\prime} F_{0}+G_{0}^{\prime} f_{0}-\frac{2 n}{n+1}\left(f_{0}^{\prime} G_{0}+F_{0}^{\prime} g_{0}\right)-$

$\frac{4 \Omega}{n+1} F_{0}^{\prime}+\frac{2 n}{n+1} \varepsilon G_{0}=0$

$\frac{1}{P r} H_{0}^{\prime \prime}+\theta_{0}^{\prime} F_{0}+H_{0}^{\prime} f_{0}+N b\left(\varnothing_{0}^{\prime} H_{0}^{\prime}+J_{0}^{\prime} \theta_{0}^{\prime}\right)+$ $2 N t \theta_{0}^{\prime} H_{0}^{\prime}+\varepsilon H_{0}=0$

$J_{0}^{\prime \prime}+S c \emptyset_{0}^{\prime} F_{0}+J_{0}^{\prime} f_{0}+\frac{N t}{N b} H_{0}^{\prime \prime}+$

$S c \varepsilon J_{0}=0$

subject to BCs

$$
\left\{\begin{array}{c}
F_{0}(0)=0, F_{0}^{\prime}(0)=0, G_{0}(0)=0, \\
H_{0}(0)=0, J_{0}(0)=0 \\
F_{0}^{\prime}(\eta) \rightarrow 0, G_{0}(\eta) \rightarrow 0 \text { as } \eta \rightarrow \infty \\
H_{0}(\eta) \rightarrow 0, J_{0}(\eta) \rightarrow 0 \text { as } \eta \rightarrow \infty
\end{array}\right.
$$

All feasible eigenvalues would be acquired $(\varepsilon)$ by solving the LEVP system. In Equation (30), a relaxed boundary condition requires being used to achieve the sequence of eigenvalues. The boundary condition $F_{0}^{\prime}(\eta) \rightarrow$ 0 as $\eta \rightarrow \infty$ is now restrained to $F_{0}^{\prime \prime}(0)$.

\section{Results and Discussions}

Non-linear Equations (8-11) subject to BCs (10) has been numerically solved with bvp4c solver in MATLAB. We have compared the values of $\sqrt{R e} C_{f x}$ and $\sqrt{R e} C_{f y}$ with the results of Zaimi et al. [48] over the stretching surface (i.e, $f^{\prime}(0)=1$ ) in Table 1 . From these results, we notice that the numerical outcomes signify good a correlation with the earlier findings. Henceforth, the code of MATLAB can be employed with full conviction to investigate the problem under discussion. The effect of numerous physical parameters such as magnetic number $(0 \leq M<0.5)$, rotation parameter $(\Omega \leq 0.04)$, positive number $(2 \leq$ $n \leq 3)$, Brownian motion parameter $(0.1 \leq$ $N b \leq 0.5)$, thermophoresis parameter $(0.1 \leq$ $N t \leq 0.5)$, and suction parameter $(S \geq 3.5)$ are conversed and illustrated in figures.

Table 1. Values of $\sqrt{\operatorname{Re}} C_{f x}$ and $\sqrt{R e} C_{f y}$ are compared when $f^{\prime}(0)=1=n$ and $M=$

\begin{tabular}{|c|c|c|c|c|}
\hline & & $\sqrt{\operatorname{Re}} C_{f x}$ & $\sqrt{\operatorname{Re}} C_{f y}$ & \\
\hline$\Omega$ & [48] & $\begin{array}{l}\text { Presen } \\
\mathrm{t} \\
\text { results }\end{array}$ & [48] & $\begin{array}{l}\text { Prese } \\
\mathrm{nt} \\
\text { results }\end{array}$ \\
\hline 0 & -1.00 & -1.000 & 0.0000 & $\begin{array}{l}0.000 \\
0\end{array}$ \\
\hline 0.5 & $\begin{array}{l}-\overline{1} \\
1.13 \\
84\end{array}$ & $\begin{array}{l}- \\
1.1384\end{array}$ & $\begin{array}{l}- \\
0.5128\end{array}$ & $\begin{array}{l}- \\
0.512 \\
8\end{array}$ \\
\hline 1 & $\begin{array}{l}-\overline{1} \\
1.32 \\
50\end{array}$ & $\begin{array}{l}- \\
1.3250\end{array}$ & $\begin{array}{l}- \\
0.8371\end{array}$ & $\begin{array}{l}- \\
0.837 \\
1\end{array}$ \\
\hline 2 & $\begin{array}{l}-\overline{1.65} \\
23\end{array}$ & $\begin{array}{l}- \\
1.6523\end{array}$ & $\overline{1}-\overline{1.2873}$ & $\begin{array}{l}- \\
1.287 \\
3\end{array}$ \\
\hline 3 & $\begin{array}{l}-\overline{1} \\
1.92 \\
89\end{array}$ & $\begin{array}{l}- \\
1.9289\end{array}$ & $\overline{1}-6248$ & $\begin{array}{l}- \\
1.624 \\
8\end{array}$ \\
\hline 4 & $\begin{array}{l}-\bar{l} \\
2.17 \\
16\end{array}$ & $\begin{array}{l}- \\
2.1716\end{array}$ & $\overline{1}-\overline{1.9054}$ & $\begin{array}{l}- \\
1.905 \\
4\end{array}$ \\
\hline 5 & $\begin{array}{l}-\overline{2} \\
2.39 \\
01\end{array}$ & $\begin{array}{l}- \\
2.3901\end{array}$ & $\overline{2} .1506$ & $\begin{array}{l}- \\
2.150 \\
6\end{array}$ \\
\hline
\end{tabular}

The existence of multiple solutions enables one to explore those parameters lead to the existence of two branches. The reduced skin friction variants $f^{\prime \prime}(0), g^{\prime}(0)$, heat transfer $-\theta^{\prime}(0)$, and $-\emptyset^{\prime}(0)$ are shown in Figures 2-5 for various values of $n$. Moreover, $S_{c}=$ $-2.40392,-2.6249,-2.8285$ is the 
Sumera Dero (et al.), Mathematical Analysis of Magnetized Rotating Nanofluid Flow Over nonlinear shrinking surface:

equivalent critic of $n=2,2.5,3$ where $S_{c}$ is the critical point where all solutions exist at $S=$ $S_{c}$. Dual branches are noted as $S \geq S_{c}$ and when $S<S_{c}$ there is no solution. The estimation of boundary layers beyond such critical values is no longer justified. Reduced skin friction $\left(f^{\prime \prime}(0)\right)$ reduces when $n$ is increased in the upper branch. Further, $g^{\prime}(0)$ decreases when $n$ increases in the lower branch. On the other hand, $g^{\prime}(0)$ rises when values of $n$ are increased in the upper branch. In addition, the behavior of $g^{\prime}(0)$ and $S$ are inversely proportional in the lower branch. Nature of reduced heat transfer $\left(-\theta^{\prime}(0)\right)$ can be seen in Figure 4 in which $-\theta^{\prime}(0)$ enhances in both branches when the effect of $S$ reduces, while the opposition movement has been examined in both branches for the rising values of $n$. Similarly, the effects of suction and positive constant were drawn in Figure 5 in order to examine their effects on the nanoparticle fraction of nanofluid. As previously noticed in Figure 4, the same behavior is noted.

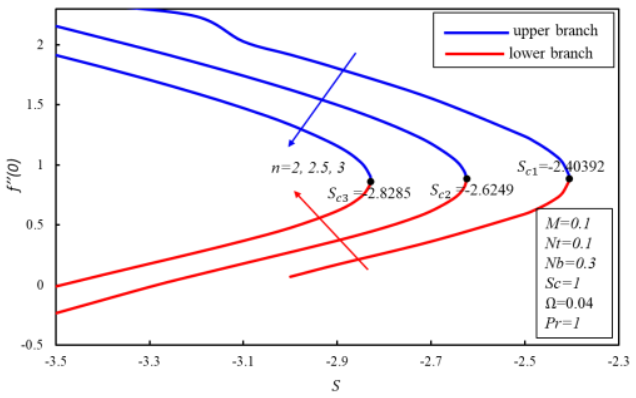

Figure 2. $f^{\prime \prime}(0)$ for numerous values of $S$ and $n$.

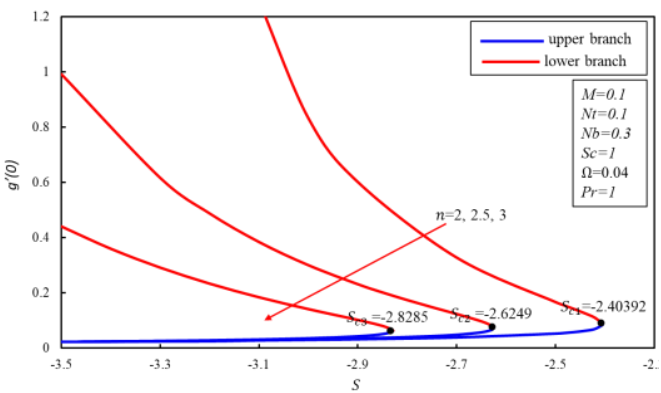

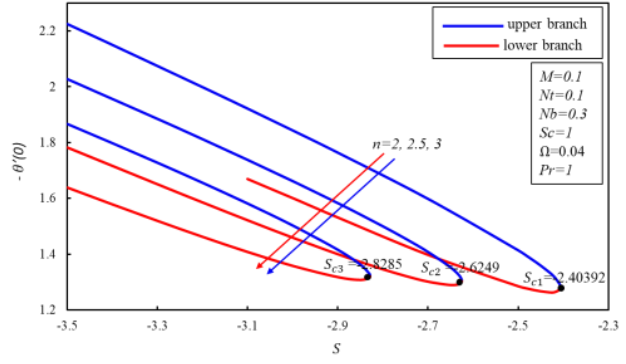

Figure 4. $-\theta^{\prime}(0)$ for numerous values of $S$ and $n$.

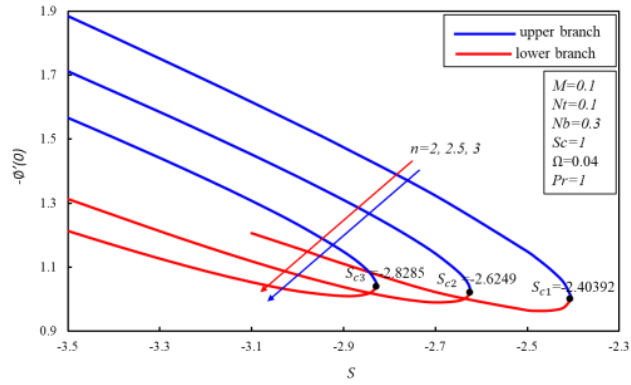

Figure 5. $-\emptyset^{\prime}(0)$ for numerous values of $S$ and $n$.

Figures 6-9 allude to the impacts of rising magnitudes of $M$ on profiles of velocity $f^{\prime}(\eta), g(\eta)$, temperature profiles $\theta(\eta)$, and concentration profiles $\varnothing(\eta)$. Figures 6 and 9 show that $f^{\prime}(\eta)$ and $g(\eta)$ decline for the rising magnitudes of $M$ in the upper solution, but the opposite movement is noticed in the lower solution. It is apparent from these estimates that for significant values of $M$, the thickness of momentum boundary layers of $f^{\prime}(\eta)$ and $g(\eta)$ are decreased in the stable branch. Physically, the decreasing behavior is due to the magnetic field effect on the nanofluid experience of the force induced by the electrical current. This electrically conductive nanofluid interacts with a transverse magnetic field that induces the Lorentz forces. The Lorentz's force reduces the velocity flow and thus reduces the thickness of layer. The temperature and concentration of nanofluid enhance in both branches when the magnetic effect increases (see Figures 8-9).

Figure 3. $g^{\prime}(0)$ for numerous values of $S$ and $n$. 

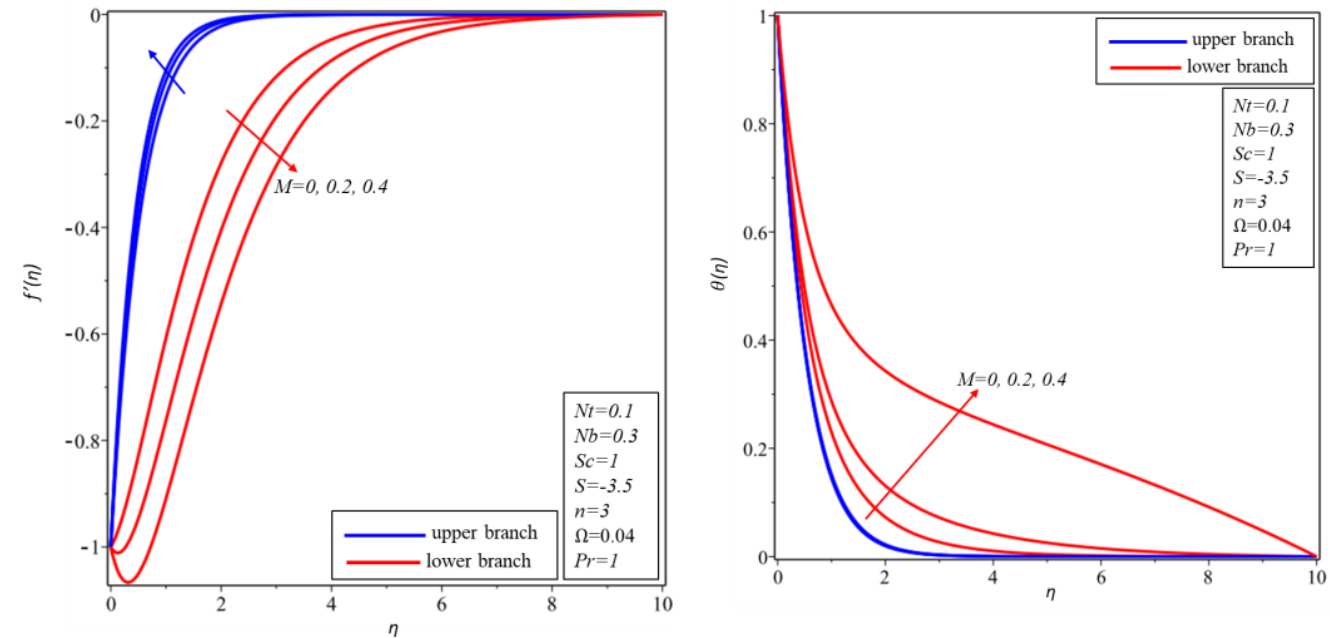

Figure 6. $f^{\prime}(\eta)$ for different values of $M$.

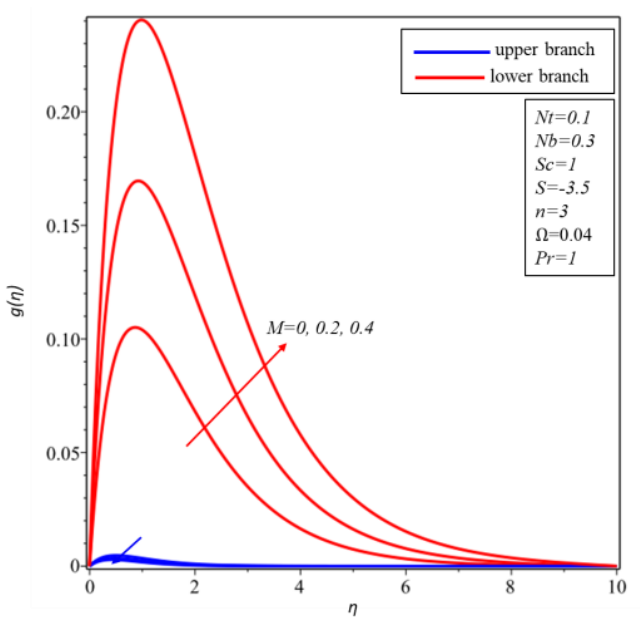

Figure 7. $g(\eta)$ for different values of $M$.

The space is intentionally left blank to adjust the fingers.
Figure 8. $\theta(\eta)$ for different values of $M$.

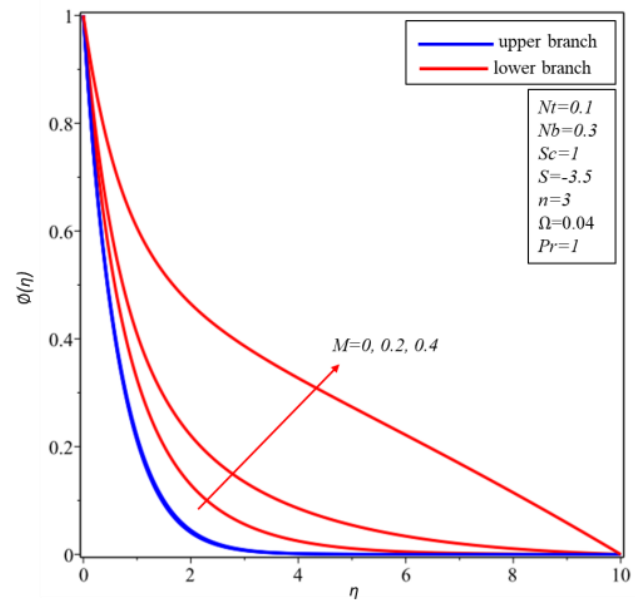

Figure 9. $\varnothing(\eta)$ for different values of $M$.

Figures 10-11 display the effect of increasing values of $N t$ on the dimensionless temperature profiles $\theta(\eta)$ and concentration profiles $\varnothing(\eta)$, respectively. These figures show that $\theta(\eta)$ and $\phi(\eta)$ increase for the increasing values of $N t$ in both branches. These increments in the thickness of boundary layers are due to the fact that the higher effect of $N t$ supports molecules of nanoparticles and fluid to transfer heat to the next layer and therefore temperature and concentration increase. 
Sumera Dero (et al.), Mathematical Analysis of Magnetized Rotating Nanofluid Flow Over nonlinear shrinking surface:

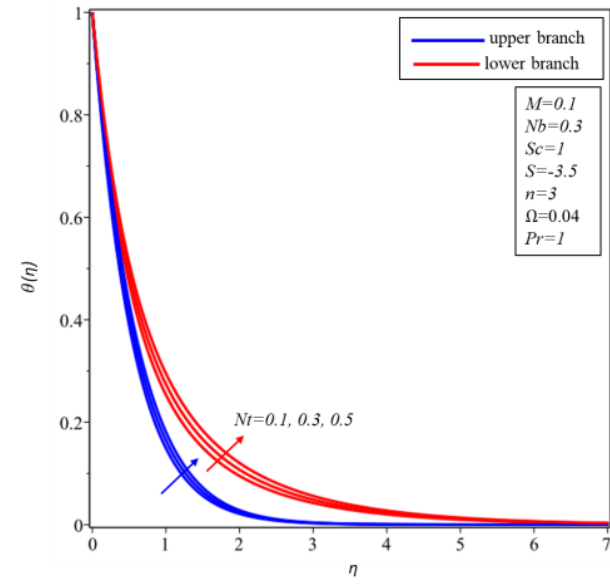

Figure 10. $\theta(\eta)$ for different values of $N t$.

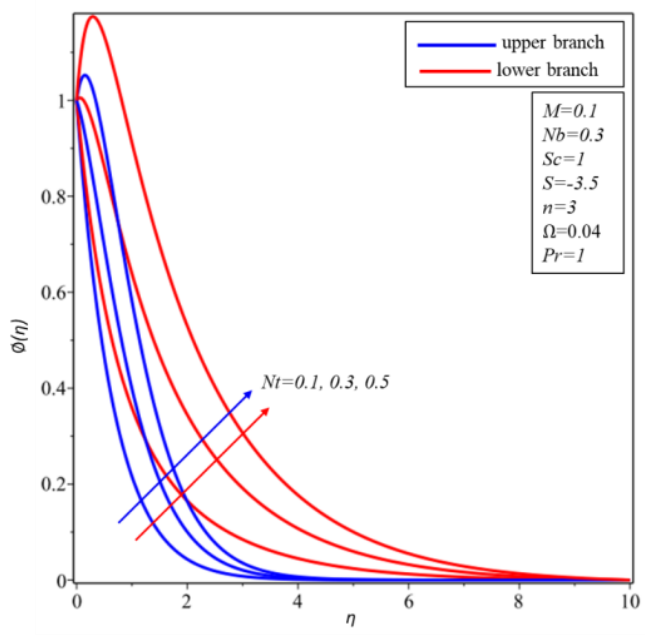

Figure 11. $\emptyset(\eta)$ for different values of $N t$.

Figures 12-13 are prepared to see the variations in temperature profiles $\theta(\eta)$ and concentration profiles $\varnothing(\eta)$ for various magnitudes of $N b$, respectively. Figure 12 indicates that $\theta(\eta)$ rises for the increasing values of $\mathrm{Nb}$ in both branches. This situation is true because, in the fluid flow process, it is possible to surge in the rate of heat transfer in the presence of the thermophoresis effect. The concentration of boundary layers, on the other hand, decreases in thickness in both branches. The consequence of Brownian motion can be described as the nanoparticles extending in the entire fluid and thus decrease in the concentration profiles.

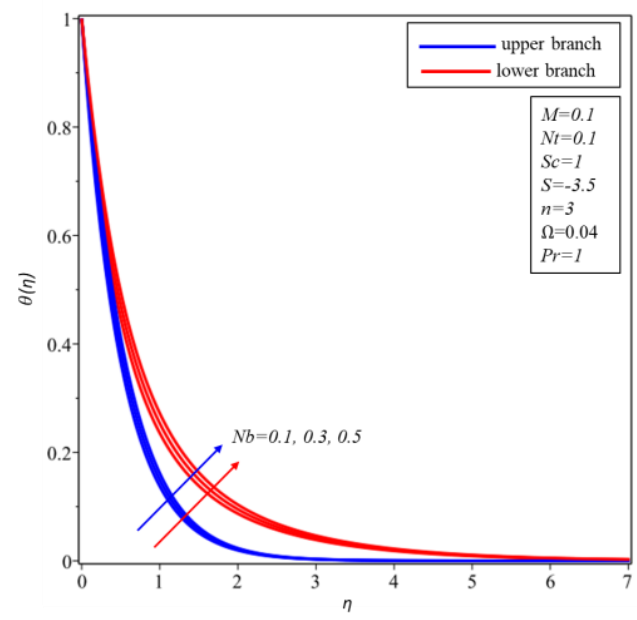

Figure 12. $\theta(\eta)$ for different values of $N b$.

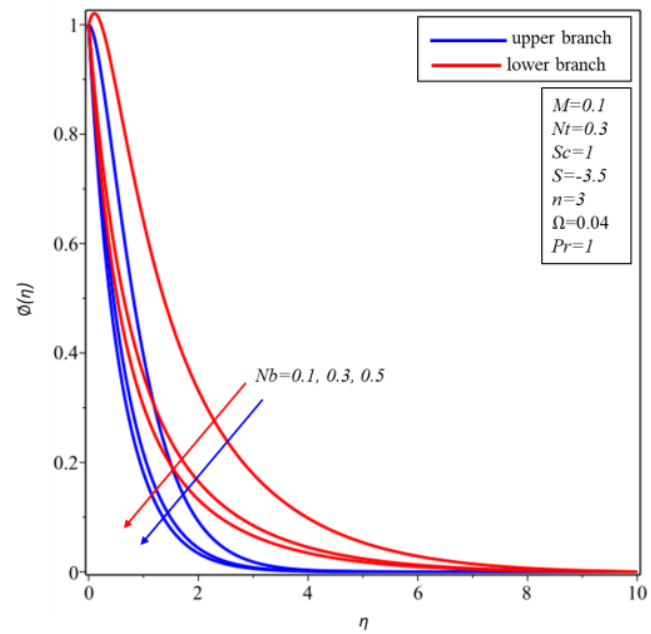

Figure 13. $\varnothing(\eta)$ for different values of $N b$.

Figure 14 illustrates that $\theta(\eta)$ decreases for increasing values of $P r$ in both branches. The development of nanofluid, a blend of center fluid and nanoparticles, depends on values of $\mathrm{Pr}$. The rising $\mathrm{Pr}$ values enhance the base fluid viscosity, resulting in a decline in the thickness of the thermal boundary layer and thus a decrease in heat transfer for the higher $P r$ values. This is because the extremely viscous nanofluid results in poor conductivities of thermal that influence the phenomenon of 
Sumera Dero (et al.), Mathematical Analysis of Magnetized Rotating Nanofluid Flow Over nonlinear shrinking surface:

conduction to reduce the thickness of thermal boundary layer. In the extremely viscous nanofluid, the motions of dispersed nanoparticles are often more impacted because of lower nanoparticle forces between one another.

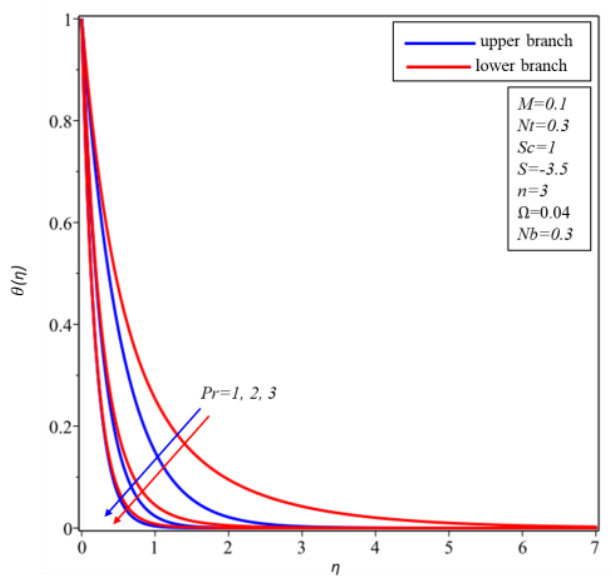

Figure 14. $\theta(\eta)$ for different values of $\operatorname{Pr}$.

Figure 15 is plotted for $\emptyset(\eta)$ to analyze the effects of $S c$. It is observed that $\varnothing(\eta)$ decreases for the increasing values of $S c$ in both branches. This decrease in $\emptyset(\eta)$ for large values of $S c$ is justified due to the fact that $S c$ is directly proportional to the kinematic viscosity of the nanofluid. The increasing values of $S c$ increase the viscosity of the nanofluid which results in a decrease in $\emptyset(\eta)$. Finally, it is noted that the stable branch has symmetrical behavior with regard to the parameter of rotation (See Figure 16). It can be easily concluded from Figure 16 that the symmetrical branches belong to this fluid model.

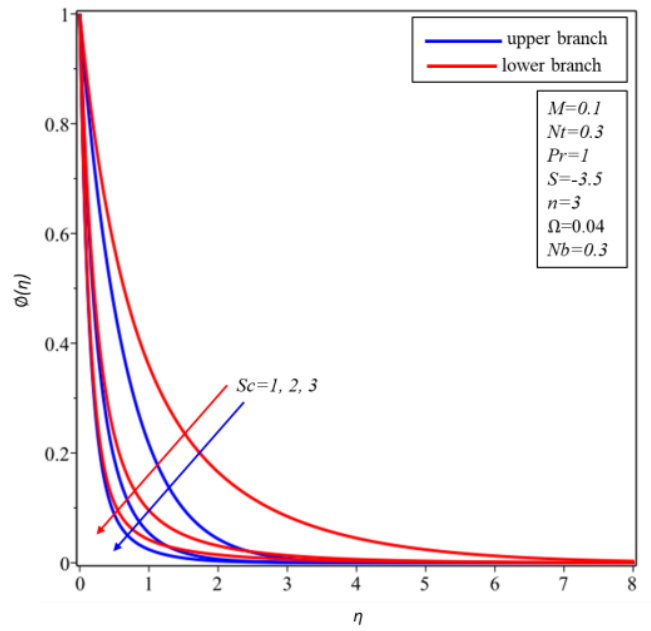

Figure 15. $\emptyset(\eta)$ for different values of $S c$.

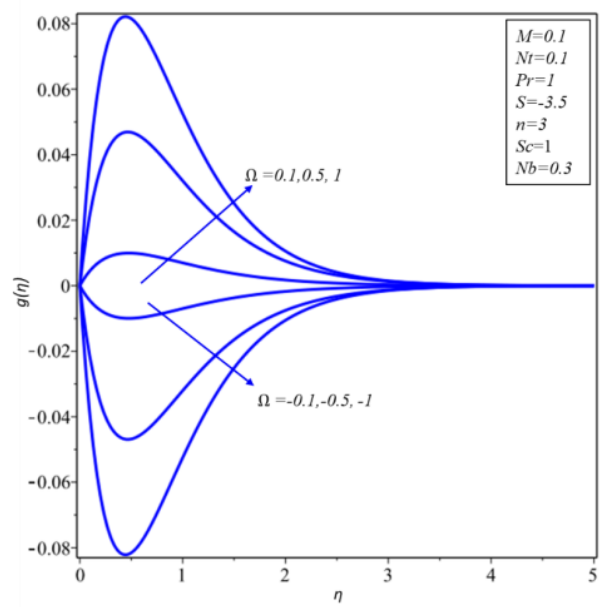

Figure 16. $g(\eta)$ for different values of $\Omega$.

Governing Equations (26-29) have been resolved by employing the bvp4c function. The results of the smallest eigenvalues are given in Table 2. The governing system provides an infinite range of eigenvalues. The smallest negative eigenvalues; $\varepsilon<0$ implies that the flow has an initial disruption development that may disrupt the flow and, ultimately, induce unstable flow. Besides that, the smallest positive eigenvalues; $\varepsilon>0$ specifies that an initial decay of disturbance occurs in the flow, are showing the stable flow. 
Sumera Dero (et al.), Mathematical Analysis of Magnetized Rotating Nanofluid Flow Over nonlinear shrinking surface:

Table 2. Values of $\varepsilon$ for $S$ and $n$ where $M=$ $0.1=N t, N b=0.3, S c=\operatorname{Pr}=1, \Omega=0.04$.

\begin{tabular}{|l|l|l|l|}
\hline$n$ & \multicolumn{1}{|c|}{$S$} & \multicolumn{1}{|c|}{$\varepsilon$} \\
\hline & & $\begin{array}{l}\text { Upper } \\
\text { branch }\end{array}$ & $\begin{array}{l}\text { Lower } \\
\text { branch }\end{array}$ \\
\hline 2 & -2.403 & 0.0001 & -0.0001 \\
\hline & -2.6 & 0.1271 & -0.0478 \\
\hline & -3 & 0.5973 & -0.8696 \\
\hline 2.5 & -2.625 & 0.0005 & -0.0007 \\
\hline & -2.8 & 0.3857 & -0.5945 \\
\hline & -3 & 0.9643 & -1.0642 \\
\hline 3 & -2.83 & 0.0002 & -0.0009 \\
\hline & -3 & 0.4585 & -0.3946 \\
\hline & -3.2 & 1.0962 & -0.9738 \\
\hline
\end{tabular}

\section{Conclusion}

In this study, we investigate MHD nanofluid 3D flow through a non-linear shrinking sheet for the heat transfer performance with multiple branches and stability analysis characteristics. The numerical analysis is conducted by applying the three-stage Labatto IIIA method in bvp4c solver to study the multiple branches of the problem with the stability analysis of the branches. Fluid suction/injection is found to have a major effect on the distribution of velocity, temperature, and concentration, which transitively influences the presence of multiple branches within the boundary layer. The main findings of the current study are

1. For $S \geq S_{c}$, there are two branches of the nanofluid problem solution, namely a lower and an upper branch. It is noticed that the lower one is not a physically suitable branch.

2. Upper branch is a stable branch from both branches.

3. An increase in the thermophoresis parameter advances nanofluid temperature along with concentration profiles.
4. Brownian motion parameter reduces the nanofluid concentration.

5. Increasing Hartmann number causes the reduction of nanofluid velocity uniformly due to the presence of Lorentz force.

\section{REFERENCES}

[1] C. Y. Wang, "Stretching a surface in a rotating fluid," Zeitschrift für angewandte Mathematik und Physik ZAMP, vol. 39, pp. 177-185, 1988.

[2] H. S. Takhar, A. J. Chamkha and Nath, "Flow and heat transfer on a stretching surface in a rotating fluid with a magnetic field". International journal of thermal sciences, vol. 42, pp. 23-31, 2003.

[3] Z. Shafique, M. Mustafa, and A. Mushtaq, "Boundary layer flow of Maxwell fluid in rotating frame with binary chemical reaction and activation energy", Results in Physics, vol. 6, pp. 627-633, 2016.

[4] A. M. Rashad, "Effects of radiation and variable viscosity on unsteady MHD flow of a rotating fluid from stretching surface in porous medium", Journal of the Egyptian Mathematical Society, vol. 22, no. 1, pp. 134142, 2014.

[5] M. Z. Ullah, A. S. Alshomrani, and M. Alghamdi, "Significance of Arrhenius activation energy in Darcy-Forchheimer 3D rotating flow of nanofluid with radiative heat transfer", Physica A: Statistical Mechanics and Its Applications, vol. 550, p. 124024, 2020.

[6] T. Hayat, A. Aziz, T. Muhammad and A, Alsaedi, "Significance of homogeneousheterogeneous reactions in DarcyForchheimer three-dimensional rotating flow of carbon nanotubes", Journal of Thermal Analysis and Calorimetry, vol. 139, no. 1, pp. 183-195, 2020.

[7] L. A. Lund, Z. Omar, S. Dero, D. Baleanu, and I. Khan, "Rotating 3D Flow of Hybrid Nanofluid on Exponentially Shrinking Sheet: Symmetrical Solution and Duality", Symmetry, vol. 12, no. 10, pp. 1637, 2020.

[8] T. Javed, M. Sajid, Z. Abbas, and N. Ali, "Non-similar solution for rotating flow over an exponentially stretching surface", International Journal of Numerical Methods for Heat \& Fluid Flow, 2011.

[9] H. Rosali, A. Ishak, R. Nazar, and I. Pop, "Rotating flow over an exponentially shrinking sheet with suction", Journal of Molecular Liquids, vol. 211, pp. 965-969, 2015. 
Sumera Dero (et al.), Mathematical Analysis of Magnetized Rotating Nanofluid Flow Over nonlinear shrinking surface: Duality and Stability

(pp. $01-13)$

[10] M. Hamid, M. Usman, T. Zubair, R. U. Haq, and W. Wang, "Shape effects of MoS2 nanoparticles on rotating flow of nanofluid along a stretching surface with variable thermal conductivity: A Galerkin approach", International Journal of Heat and Mass Transfer, vol. 124, pp. 706-714, 2018.

[11] N. Faraz, and Y. Khan, "Analytical solution of electrically conducted rotating flow of a second grade fluid over a shrinking surface", Ain Shams Engineering Journal, vol. 2, no. 3-4, pp. 221-226, 2011.

[12] M. Khan, A. Hafeez, and J. Ahmed, "Impacts of non-linear radiation and activation energy on the axisymmetric rotating flow of OldroydB fluid", Physica A: Statistical Mechanics and Its Applications, p. 124085, 2020.

[13] B. B. William, "Magnetohydrodynamichypersonic flow past a blunt body", Journal of the Aerospace Sciences, vol. 25, no. 11, pp. 685-690, 1958

[14] B. B. William "The stagnation-point boundary layer in the presence of an applied magnetic field", Journal of the Aerospace Sciences, vol. 28, no. 8, pp. 610-611, 1961.

[15] T. E. Eastman, E. W. Hones, S. J. Bame, and J. R. Asbridge, "The magnetospheric boundary layer: Site of plasma, momentum and energy transfer from the magnetosheath into the magnetosphere", Geophysical research letters, vol. 3 , no. 11, pp. 685-688, 1976.

[16] M. A. Hossain, "Viscous and Joule heating effects on MHD free convection flow with variable plate temperature (No. IC--90/265) ", International Centre for Theoretical Physics, 1990.

[17] V. Malapati and P. Polarapu, "Unsteady MHD free convective heat and mass transfer in a boundary layer flow past a vertical permeable plate with thermal radiation and chemical reaction", Procedia Engineering, vol. 127, pp. 791-799, 2015.

[18] S. K. Soid, A. Ishak, and I. Pop, "MHD flow and heat transfer over a radially stretching/shrinking disk", Chinese Journal of Physics, vol. 56, no. 1, pp. 58-66, 2018.

[19] K. L. Hsiao, "Micropolar nanofluid flow with MHD and viscous dissipation effects towards a stretching sheet with multimedia feature", International Journal of Heat and Mass Transfer, vol. 112, pp. 983-990, 2017.

[20] J. Buongiorno, "Convective transport in nanofluids", Journal of heat transfer, vol. 128, no. 3, pp. 240-250, 2006.

[21] S. Dero, A. M. Rohni, and A. Saaban, "MHD micropolar nanofluid flow over an exponentially stretching/shrinking surface:
Triple solutions", J. Adv. Res. Fluid Mech.

Therm. Sci, vol. 56, pp. 165-174, 2019.

[22] L. A. Lund, Z. Omar, I. Khan, E. S. M. Sherif, and H. S. Abdo, "Stability analysis of the magnetized casson nanofluid propagating through an exponentially shrinking/stretching plate: dual solutions", Symmetry, vol. 12, no. 7, p. 1162, 2020.

[23] G. Rasool, A. Shafiq, and D. Baleanu, "Consequences of Soret-Dufour Effects, Thermal Radiation, and Binary Chemical Reaction on Darcy Forchheimer Flow of Nanofluids", Symmetry, vol. 12, no. 9, p. 1421, 2020.

[24] A. Dawar, Z. Shah, P. Kumam, H. Alrabaiah, W. Khan, S. Islam, and N. Shaheen, "Chemically reactive MHD micropolar nanofluid flow with velocity slips and variable heat source/sink", Scientific Reports, vol. 10, no. 1, pp. 1-23, 2020.

[25] Z. Khan, H. U. Rasheed, T. Abbas, W. Khan, I. Khan, D. Baleanu, and K. Sooppy Nisar, "Analysis of Eyring-Powell Fluid Flow Used as a Coating Material for Wire with Variable Viscosity Effect along with Thermal Radiation and Joule Heating", Crystals, vol. 10, no. 3, p. 168, 2020.

[26] N. R. Bala, S. K. Bala, L. K. Saha, and M. A. Hossain, "Influence of undulating wall heat and mass flux on MHD natural convection boundary layer flow from a vertical wall", Heat Transfer, 2020.

[27] U. Rashid, D. Baleanu, A. Iqbal, and M. Abbas, "Shape Effect of Nanosize Particles on Magnetohydrodynamic Nanofluid Flow and Heat Transfer over a Stretching Sheet with Entropy Generation", Entropy, vol. 22, no. 10, p. $1171,2020$.

[28] M. M. Rashidi, N. V. Ganesh, A. A. Hakeem, and B. Ganga, "Buoyancy effect on MHD flow of nanofluid over a stretching sheet in the presence of thermal radiation", Journal of Molecular Liquids, vol. 198, pp. 234-238, 2014.

[29] R. Gentle, P. Edwards, and W. Bolton, "Mechanical engineering systems", Elsevier, 2001.

[30] L. A. Lund, Z. Omar, S. Dero, I. Khan, D. Baleanu, and K. S. Nisar, "Magnetized flow of $\mathrm{Cu}+\mathrm{A} 12 \mathrm{O} 3+\mathrm{H} 2 \mathrm{O}$ hybrid nanofluid in porous medium: Analysis of duality and stability", Symmetry, vol. 12, no. 9, p. 1513, 2020.

[31] M. K. Nayak, "MHD 3D flow and heat transfer analysis of nanofluid by shrinking surface inspired by thermal radiation and viscous dissipation", International Journal of Mechanical Sciences, vol. 124, pp. 185-193, 2017. 
[32] N. A. L. Aladdin, N. Bachok, and N. S. Anuar, "MHD Stagnation Point Flow in Nanofluid Over Shrinking Surface Using Buongiorno's Model: A Stability Analysis", Journal of Advanced Research in Fluid Mechanics and Thermal Sciences, vol. 76, no. 3, pp. 12-24, 2020.

[33] M. R. Khan, "Numerical analysis of oblique stagnation point flow of nanofluid over a curved stretching/shrinking surface", Physica Scripta, vol. 95, no. 10, p. 105704, 2020.

[34] N. S. Khashi'ie, N. M. Arifin, and I. Pop, "Unsteady axisymmetric flow and heat transfer of a hybrid nanofluid over a permeable stretching/shrinking disc", International Journal of Numerical Methods for Heat \& Fluid Flow, 2020.

[35] M. I. Afridi, T. A. Alkanhal, M. Qasim, and I. Tlili, "Entropy Generation in $\mathrm{Cu}-\mathrm{Al} 2 \mathrm{O} 3-\mathrm{H} 2 \mathrm{O}$ Hybrid Nanofluid Flow over a Curved Surface with Thermal Dissipation", Entropy, vol. 21, no. 10, p. 941, 2019.

[36] H. Masuda, A. Ebata, and K. Teramae, "Alteration of thermal conductivity and viscosity of liquid by dispersing ultra-fine particles. Dispersion of $\mathrm{Al} 2 \mathrm{O} 3, \mathrm{SiO} 2$ and $\mathrm{TiO} 2$ ultra-fine particles", Netsu Bussei(Japan), vol. 4, pp. 227-233, 1993.

[37] M. Abareshi, E. K. Goharshadi, S. M. Zebarjad, H. K. Fadafan, and A. Youssefi, "Fabrication, characterization and measurement of thermal conductivity of Fe3O4 nanofluids", Journal of Magnetism and Magnetic Materials, vol. 322, no. 24, pp. 3895-3901, 2010.

[38] S. K. Das, N. Putra, P. Thiesen, and W. Roetzel, "Temperature dependence of thermal conductivity enhancement for nanofluids", Journal of heat transfer, vol. 125, no. 4, pp. 567-574, 2003.

[39] S. K. Das, S. U. Choi, W. Yu, and T. Pradeep, "Nanofluids: science and technology", New Jersey: John Wiley \& Sons, 2007.

[40] B. C. Sakiadis, "Boundary-layer behavior on continuous solid surfaces: I. Boundary-layer equations for two-dimensional and axisymmetric flow", AIChE Journal, vol. 7, no. 1, pp.26-28, 1961.

[41] B. C. Sakiadis, "Boundary-layer behavior on continuous solid surfaces: II. The boundary layer on a continuous flat surface", AiChE journal, vol. 7, no. 2, pp. 221-225, 1961.

[42] A. M. Rohni, S. Ahmad, and I. Pop, "Flow and heat transfer at a stagnation-point over an exponentially shrinking vertical sheet with suction", International journal of thermal sciences, vol. 75, pp. 164-170, 2014.

[43] L. J. Crane, "Flow past a stretching plate", Zeitschrift für angewandte Mathematik und Physik ZAMP, vol. 21, no. 4, pp. 645647, 1970.

[44] I. S. Awaludin, A. Ishak, and I. Pop, "Stagnation Point Flow and Heat Transfer Over a Permeable Stretching/Shrinking Sheet with Heat Source/Sink", In International Conference on Computational \& Experimental Engineering and Sciences, pp. 189-199. 2019.

[45] J. Raza, S. Dero, L. A. Lund, and Z. Omar, "Duality and stability of MHD DarcyForchheimer porous medium flow of rotating nanofluid on a linear shrinking/stretching sheet: Buongiorno model", International Journal of Numerical Methods for Heat \& Fluid Flow, 2021.

[46] L. A. Lund, Z. Omar, I. Khan, and E. S. M. Sherif, "Dual Branches of MHD ThreeDimensional Rotating Flow of Hybrid Nanofluid on Nonlinear Shrinking Sheet", Computers Materials \& Continua, vol. 66, no. 1, pp. 127-139, 2021.

[47] J. H. Merkin, "On dual solutions occurring in mixed convection in a porous medium", Journal of Engineering Mathematics, vol. 20 no. 2, pp. 171-179, 1986.

[48] K. Zaimi, A. Ishak, and I. Pop, "Stretching surface in rotating viscoelastic fluid", Applied Mathematics and Mechanics, vol. 34, no. 8, pp. 945-952, 2013. 